\section{Commentary: Patch repair for aortomitral endocarditis: Playing the short game or the long game?}

\author{
Sameer A. Hirji, MD, MPH, and \\ Tsuyoshi Kaneko, MD
}

Aortomitral infectious endocarditis (IE) remains a challenging problem in cardiac surgery from both technical and management standpoints. Because there are no standardized approaches to the definitive management of aortomitral IE, most procedures often entail nuanced approaches to reconstruction, depending on the extent of IE involvement.

That said, we read with great interest the case report by Benedetto and colleagues ${ }^{1}$ in this issue of the Journal. The authors demonstrate a unique concomitant use of the Ozaki procedure for the management of aortic valve IE and transaortic approach to mitral valve repair (MVr) using the autologous pericardium for management of anterior mitral valve leaflet perforation. They are to be congratulated for their outcomes and their innovative approach in a relatively low-risk (EuroScore II, 2.4\%), healthy, 50-year-old male.

This case highlights several key advantages. First, Ozaki's autologous pericardium aortic valve neo-cuspidization (AVNeo) procedure helped avoid aortic root replacement using homografts. This alternative approach helps minimize extensive surgical trauma and the inherent risks associated with bleeding in the setting of IE-induced coagulopathy. ${ }^{2,3}$ The use of an aortic valve replacement with biosprosthesis would have been a comparable alternative, but the incidence rate of IE necessitating reoperation

From the Division of Cardiac Surgery, Department of Surgery, Brigham and Women's Hospital, Harvard Medical School, Boston, Mass.

Disclosures: Dr Kaneko has served as a consultant/speaker for Edwards Lifesciences, Medtronic, Abbott Structural Heart, Baylis Medical, and 4C Medical. Dr Hirji reported no conflicts of interest.

The Journal policy requires editors and reviewers to disclose conflicts of interest and to decline handling or reviewing manuscripts for which they may have a conflict of interest. The editors and reviewers of this article have no conflicts of interest.

Received for publication May 4, 2020; revisions received May 4, 2020; accepted for publication May 8, 2020; available ahead of print May 16, 2020.

Address for reprints: Tsuyoshi Kaneko, MD, Division of Cardiac Surgery, Brigham and Women's Hospital, 15 Francis St, Boston, MA, 02115 (E-mail: tkaneko2@ bwh.harvard.edu).

JTCVS Techniques 2020;3:104-5

2666-2507

Copyright $(2020$ The Authors. Published by Elsevier Inc. on behalf of The American Association for Thoracic Surgery. This is an open access article under the CC BY-NC-

ND license (http://creativecommons.org/licenses/by-nc-nd/4.0/).

https://doi.org/10.1016/j.xjtc.2020.05.007

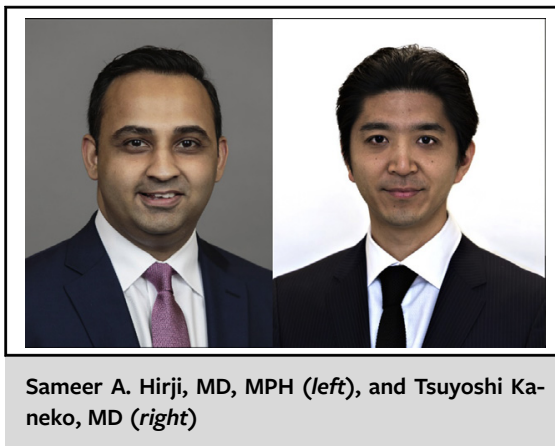

CENTRAL MESSAGE

Combined autologous pericar-

dium aortic valve neocuspidiza-

tion and transaortic mitral valve

repair may be an attractive short-

term strategy for aortomitral

endocarditis, but long-term

durability remains to be

determined.

is much higher compared with the Ozaki procedure ( $\sim 1.0 \%$ vs $0.3 \%$ per patient-year). $)^{3,4}$ The use of fresh autologous pericardium for MVr further reduced the potential future risk of reinfection due to the presence of prosthetic material. ${ }^{5}$ Second, this case avoided the conventional approach to MVr via the left atriotomy. Although the exact benefits of this strategy remain unknown, in theory, avoidance of left atriotomy may further minimize the need for additional incisions. Instead, the transaortic approach facilitated convenient exposure of the anterior mitral valve leaflet for reconstruction through the single exposure via the aorta.

Although the utility of the combined AVNeo procedure and transaortic MVr may represent an attractive short-term strategy and is appropriately justified, there are key longterm issues to consider. First, knowledge of the longevity and subsequent risk of reinfection following this combined procedure is limited, given that the longest reported series with the Ozaki procedure has a 10-year follow-up, with cumulative incidences of reoperation and recurrent aortic regurgitation of $4.2 \%$ and $7.3 \%$, respectively. ${ }^{3}$ Second, the feasibility of future valve-in-valve transcatheter aortic valve options may be questionable with autologous patching, although the exact feasibility is not known at this time. Finally, the high mitral regurgitation recurrence rate with autologous patching in the absence of annular ring is 
another concern. Thus, as the durability of this combined approach remains to be determined, we suspect that this patient will most likely require a subsequent future reoperation, given his young age.

Because the long-term performance of the Ozaki procedure in terms of hemodynamics and ease of subsequent valve implantation is not well elucidated, long-term follow-up studies are warranted before this technique becomes routine practice. This begs the question as to whether we are playing the short game or the long game when it comes to dealing with aortomitral endocarditis.

\section{References}

1. Benedetto U, Gergely S, Dimagli A, Sinha S. AVNeo (Ozaki) and transaortic mitral valve repair using the autologous pericardium only for aortomitral endocarditis. J Thorac Cardiovasc Surg Tech. 2020;3:101-3.

2. Goldstone AB, Woo YJ. Alternative approaches for mitral valve repair. Ann Cardiothorac Surg. 2015;4:469-73.

3. Ozaki S, Kawase I, Yamashita H, Uchida S, Takatoh M, Kiyohara N. Midterm outcomes after aortic valve neocuspidization with glutaraldehyde-treated autologous pericardium. J Thorac Cardiovasc Surg. 2018;155:2379-87.

4. Lengyel M, Janosi A, Arvay A. The incidence of endocarditis caused by a prosthetic valve and its risk factors [in Hungarian]. Orv Hetil. 1989;130:765-72.

5. Quinn RW, Wang L, Foster N, Pasrija C, Ghoreishi M. Long-term performance of fresh autologous pericardium for mitral valve leaflet repair. Ann Thorac Surg. 2020;109:36-41. 